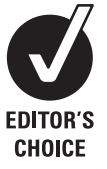

See Editorial Commentary, p 939

${ }^{1}$ Movement Disorders,

Functional Neurosurgery and Neurophysiology Units, Centro Internacional de Restauración Neurológica (CIREN), La Habana, Cuba; ${ }^{2}$ Department of

Neurology and Neurosurgery, Clínica Universitaria and Medical School and Neuroscience Division, CIMA, University of

Navarra, Pamplona, Spain;

${ }^{3}$ Centro de Investigación

Biomédica en Red sobre

Enfermedades

Neurodegenerativas (CIBERNED), Pamplona, Spain;

${ }^{4}$ Department of Neurology, Emory University School of Medicine, Atlanta, Georgia, USA

Correspondence to:

Professor J A Obeso, Clínica

Universitaria, Pio XII Avenue, 36,

31008 Pamplona, Spain;

jobeso@unav.es

Received 30 June 2008

Revised 7 November 2008

Accepted 25 November 2008

Published Online First

9 February 2009

\title{
Therapeutic efficacy of unilateral subthalamotomy in Parkinson's disease: results in 89 patients followed for up to 36 months
}

\author{
L Alvarez, ${ }^{1}$ R Macias, ${ }^{1}$ N Pavón, ${ }^{1}$ G López, ${ }^{1}$ M C Rodríguez-Oroz, ${ }^{2,3}$ R Rodríguez, ${ }^{1}$ \\ M Alvarez, ${ }^{1}$ I Pedroso, ${ }^{1} \mathrm{~J}$ Teijeiro, ${ }^{1}$ R Fernández, ${ }^{1}$ E Casabona, ${ }^{1}$ S Salazar, ${ }^{1}$ C Maragoto, ${ }^{1}$ \\ M Carballo, ${ }^{1}$ I García, ${ }^{1}$ J Guridi, ${ }^{2,3} \mathrm{~J}$ L Juncos, ${ }^{4}$ M R DeLong, ${ }^{4} \mathrm{~J}$ A Obeso ${ }^{2,3}$
}

\begin{abstract}
Background: Stereotactic thermocoagulative lesions of the subthalamic nucleus (STN) have been shown to induce significant motor improvement in patients with Parkinson's disease (PD).
\end{abstract}

Patients and methods: 89 patients with PD were treated with unilateral subthalamotomy. 68 patients were available for evaluations after 12 months, 36 at 24 months and 25 at 36 months.

Results: The Unified Parkinson's Disease Rating Scale (UPDRS) motor scores improved significantly contralaterally to the lesion in the "off" and "on" states throughout the follow-up, except for the "on" state at the last evaluation. Axial features and signs ipsilateral to the lesion progressed steadily throughout the study. Levodopa daily doses were significantly reduced by $45 \%, 36 \%$ and $28 \%$ at 12, 24 and 36 months post-surgery. 14 patients (15\%) developed postoperative hemichorea-ballism which required pallidotomy in eight. These 14 patients had significantly higher dyskinesia scores (levodopa induced) preoperatively than the entire cohort.

Conclusion: Unilateral subthalamotomy was associated with significant and sustained motor benefit contralateral to the lesion. Further work is needed to ascertain what factors led to severe, persistent chorea-ballism in a subset of patients. Subthalamotomy may be considered an option in circumstances when deep brain stimulation is not viable.

Deep brain stimulation (DBS) of the subthalamic nucleus (STN) or globus pallidus pars interna has shown significant and sustained benefit in patients with advanced Parkinson's disease (PD) and is the current treatment of choice worldwide. Hyperactivity of the STN results in fundamental pathophysiological changes experimentally and in patients with PD. ${ }^{1-4}$ The beneficial effects of blocking or lesioning the STN in MPTP monkeys $^{5-7}$ played a decisive role in revitalising surgery as a practical therapy for $\mathrm{PD}$. Occasionally, ablative surgery instead of DBS may be necessary. This may occur, for instance, when the DBS device has to be removed due to persistent infection or some technical problem. DBS may not be easily applied in patients living in remote regions or may be contraindicated in patients suffering from immune deficiency or personality disorders. Finally, the cost of DBS and its associated consumables is not affordable for many patients in countries without a strong public health care system.
We argued several years ago that a lesion of the STN could be performed in patients with PD with a low risk of inducing severe hemichorea-ballism, ${ }^{8}$ a potential surgical complication regarded as likely with any lesion of the STN in normal individuals or primates. The assumption that the results of such lesions would behave differently was based on experimental findings in normal and MPTP intoxicated monkeys, ${ }^{7}$ and on data from the classic stereotactic surgical literature of the 1960s and 1970s in patients with PD. The latter indicated a low incidence of dyskinesias when the STN was lesioned accidentally during surgery. ${ }^{9}{ }^{10}$

Two initial independent pilot studies in small samples of patients indicated that unilateral subthalamotomy could be performed safely in most patients. ${ }^{11}{ }^{12}$ Subsequently, our group ${ }^{13}$ and others $^{14-16}$ have reported a significant anti-PD benefit contralateral to STN lesions for up to 12 months postoperatively. We also described 18 patients in whom bilateral subthalamotomy was associated with a good benefit to risk ratio. ${ }^{17}$ Nonetheless, the irreversible nature of the lesion and the possibility of inducing hemichorea-ballism remain legitimate concerns that reduce the appeal and practical applicability of subthalamotomy in PD.

Here, we describe a series of 89 patients with $\mathrm{PD}$ treated with unilateral subthalamotomy over a decade and followed for up to 36 months. This is the largest series of patients with the longest postoperative follow-up reported to date. It thus provides the most accurate estimate so far of the risks and therapeutic value of subthalamotomy in PD.

\section{PATIENTS AND METHODS}

The study protocol was approved by the institutional scientific committee and the Cuban National Ethics Committee, and was undertaken according to the Declaration of Helsinki. All patients gave informed consent to participate in the study. Patients were recruited and operated on in the Centro Internacional de Restauracion Neurologica (CIREN), Havana, Cuba. Patients fulfilled the UK Parkinson's Disease Society Brain Bank diagnostic criteria ${ }^{18}$ and suffered motor fluctuations with disabling "off" episodes (scores $>35$ on the Unified Parkinson's Disease Rating Scale (UPDRS)) and dyskinesias that could not be adequately controlled pharmacologically. They 
Table 1 Clinical demographics of patients treated with unilateral subthalamotomy

\begin{tabular}{ll}
\hline Characteristic & \\
\hline Age (years) & $56.9(40-73)$ \\
Sex (men/women) & $61 / 7$ \\
Disease duration (years) & $10.8(5-27)$ \\
Levodopa daily dose (mg) & $1085.8(600-2000)$ \\
No of doses/day & $5.4(4-8)$ \\
Hoehn and Yahr† & \\
II & 10 \\
III & 54 \\
IV & 25 \\
UPDRS part III in "off" state & $66.8(35-83)$ \\
UPDRS part III in "on" state & $30.2(7-40)$ \\
\hline
\end{tabular}

Data are presented as mean (range) or number.

*Levodopa equivalents calculated as $10 \mathrm{mg}$ of bromocriptine $=1 \mathrm{mg}$ pergolide $=100 \mathrm{mg}$ levodopa.

tHoenh and Yahr scale in the "off" medication state.

UPDRS, Unified Parkinson's Disease Rating Scale.

were recruited consecutively to the surgical protocol and were all operated on from 1995 to 2004; this study thus includes the 11 patients reported previously by this team. ${ }^{13}$ The demographic characteristics of this cohort of patients with PD are summarised in table 1. All patients were treated chronically with levodopa and benserazide, and most also received a dopamine agonist (bromocriptine or pergolide). Patients suffering from severe (grade IV, see scoring scale below) "peak of dose" or diphasic dyskinesias were not treated with subthalamotomy but referred for pallidotomy when appropriate.

\section{Surgical technique}

Details of the procedure have been reported elsewhere. ${ }^{13} 171920$ Surgery was performed under local anaesthesia. A series of parallel axial slices were obtained by CT to identify the anterior and posterior commissures. Coordinates for targeting the STN were $13 \mathrm{~mm}$ lateral, $2 \mathrm{~mm}$ behind the midpoint and $4 \mathrm{~mm}$ below the intercommissural plane. The trajectory to the target was defined using a software program for surgical planning system (STASSIS). ${ }^{21}$ Semi-microrecording of multiunit action potentials and online integration of the amplitude and number of spikes at the recording sites were used to localise the STN applying the customised program Neuro-restorative Deep Recording System. ${ }^{22}$ The mean number of unilateral recording tracks per patient was 5.6 (range 3-14). Stimulation through the recording electrode $(60-180 \mathrm{~Hz}, 0.1-5 \mathrm{~mA}$ and $0.3 \mathrm{~ms}$ pulse width) was used to look for therapeutic benefit and possible side effects such as dyskinesias, sensory or motor responses, oculomotor effects, etc. The thermolytic lesion was applied using a radiofrequency probe of $1.1 \mathrm{~mm}$ diameter and a 2 or $4 \mathrm{~mm}$ exposed tip (Elekta Instruments $\mathrm{AB}$, Sweden). The region to be lesioned was first warmed to $42^{\circ} \mathrm{C}, 50^{\circ} \mathrm{C}$ and $60^{\circ} \mathrm{C}$ for $10 \mathrm{~s}$ periods separated by 1-2 min intervals. Subsequently, a lesion was performed with a power of $8 \mathrm{~W}$ at $70^{\circ} \mathrm{C}$ for $60 \mathrm{~s}$. The lesion was intended to impinge mainly on the dorsolateral region of the STN with a volume of approximately $40-60 \mathrm{~mm}^{3}$ (fig 1). In most patients, the lesion extended about $0.5-1 \mathrm{~mm}$ above the dorsal boundary of the STN. The lesion was performed contralaterally to the clinically most affected side. This was the right hemisphere in 41 patients and the left hemisphere in 48 patients. A routine CT brain scan was performed 24-48 h postoperatively in all patients. Patients remained under surveillance in the neurosurgical unit for $24 \mathrm{~h}$ and in hospital for a total of 7-12 days. The location of the lesion was subsequently ascertained by MRI (Siemens Symphony $1.5 \mathrm{~T}$ ) in 56 patients postoperatively and repeated after $2-3$ years (fig 2 ) in 32 patients.

\section{Assessment}

Patients were assessed 1-4 weeks before surgery and at 1 and 6 months during the first year after operation, and annually thereafter for up to 36 months. These annual evaluations were conducted by the neurologists regularly attending patients with $\mathrm{PD}$ in the CIREN and by neurologists from other centres participating in this project. The CAPIT guidelines were followed for clinical assessment. ${ }^{23}$ The UPDRS part II (activities of daily living) and part III (motor part) and a revised version of the CAPIT dyskinesias scale were used for evaluations. ${ }^{24}$ The latter scale rates the intensity and duration of dyskinesias from 0 (absent) to 4 (very severe, continuous and generalised) and scores separately "off" period dystonia, diphasic and "on" dyskinesias. In each evaluation, patients were scored in the "off" state after a minimum of $12 \mathrm{~h}$ overnight without medication. The assessment was repeated in the "on" state following 200/50 mg of levodopa/benserazide or 250/25 mg of levodopa/carbidopa at 09:00 in the fasting state. A battery of neuropsychological tests was administered to assess cognitive status before and after operation (12 and 24 months) in 33 patients. These patients were not selected according to any specific clinical feature or the outcome of surgery but were the ones available for such assessment over prolonged follow-up.

Evaluations included the Mini-Mental State Examination (normal 30), Rey-Osterreich test for visuo-constructive functions (normal 18-36), the frontal assessment battery (normal 14-18), verbal fluency (Mattis supermarket, minimum 20 words
Figure 1 Examples of a lesion of approximately average size $\left(37 \mathrm{~mm}^{3}\right)(\mathrm{A})$ for the cohort and a large $\left(90 \mathrm{~mm}^{3}\right)(B)$ lesion as shown by MRI taken during the first week after surgery.
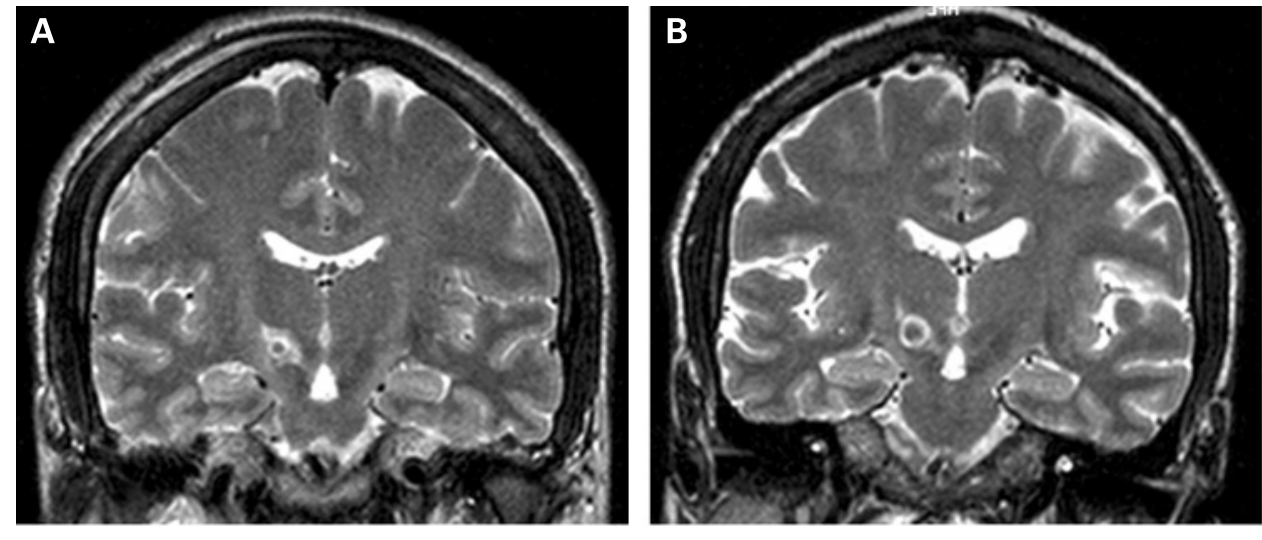
Figure $2 \mathrm{MRI}$ showing the evolution of the image associated with a thermolytic lesion in the right subthalamic nucleus. The acute lesion (B) placed in the dorsolateral region of the nucleus starts to decrease at 1 month $(C)$ and is drastically shrunk at 6 months (D). However, the lesion is still recognisable at 6 months compared with the preoperative $\mathrm{MRI}$ image (A).
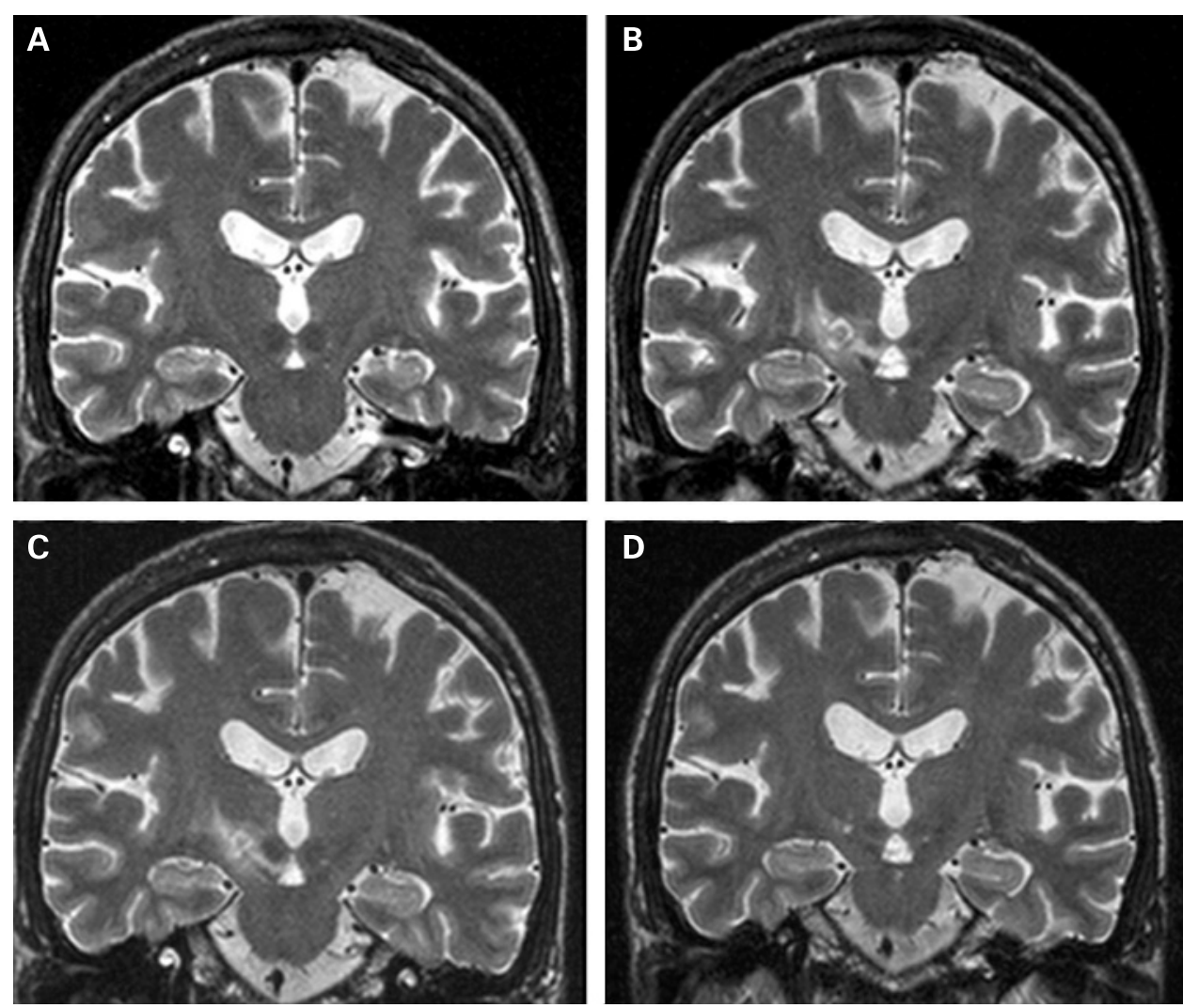

per minute) and Hamilton Depression Score $(<12)$, all of which we use routinely to assess the effect of surgery in PD. ${ }^{17} 25$

\section{Statistical analysis}

The primary outcome measurement was the change induced by unilateral subthalamotomy in the UPDRS part III (motor) in

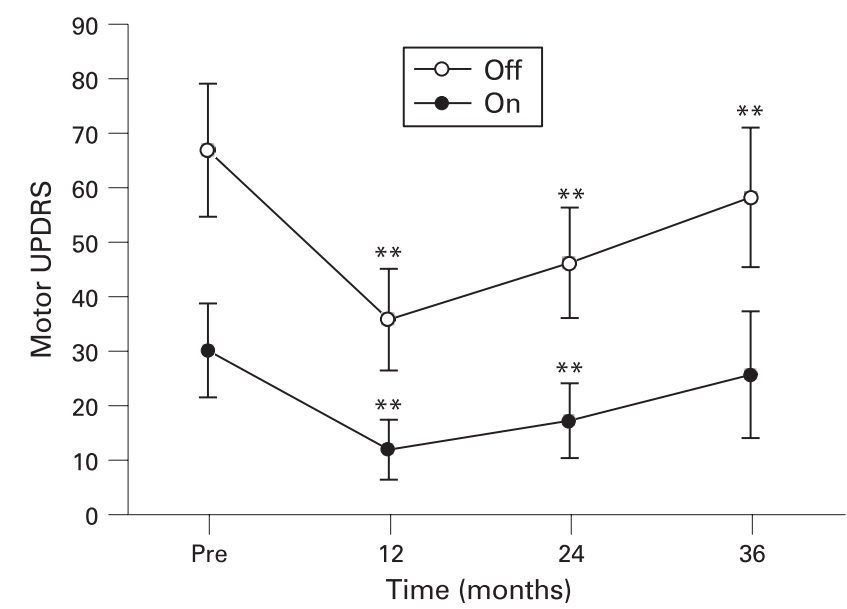

$\begin{array}{lcccc}\text { L-DOPA } & 1085.8 & 589.2 & 693.0 & 779.5 \\ (\mathrm{mg} / \text { day }) & (600-2000) & (200-1200) & (300-1000) & (400-1200)\end{array}$

Figure 3 Long term effect of unilateral subthalamotomy on the Unified Parkinson's Disease Rating Scale (UPDRS) motor in the "off" and "on" motor states. Preoperative (baseline, $n=89$ ), 12 months postoperatively $(n=68), 24$ months postoperatively $(n=36)$ and 36 months postoperatively $(n=25)$. ${ }^{* *}$ Significant differences between pre and postoperative scores $(p<0.01$, Wilcoxon's matched paired test). Mean (range) levodopa dose equivalents per day for each evaluation are given at the bottom. the "off" state at the time of the last evaluation (ie, 3 years) compared with the pre-surgery score. Secondary endpoints included the effect of subthalamotomy in the following items: (i) UPDRS part III subscores for bradykinesia, rigidity, tremor (cardinal features) in the off state; (ii) UPDRS part III in the "on" medication state; (iii) levodopa daily dose equivalents; and (iv) modification of dyskinesia score. Additional secondary endpoints were differences in the UPDRS part III in the "off" medication state at 12 and 36 months and modifications in the battery of neuropsychological tests 2 years after surgery.

The Friedman test was applied for repeated measurements of the effect of subthalamotomy on the various outcome variables, and Bonferroni's correction was applied for pairwise comparisons. The Wilcoxon test was used for paired evaluations. The significance level was $\mathrm{p}<0.05$.

\section{RESULTS}

Sixty-eight patients were assessed at 12 months, 36 patients at 24 months and 25 at 36 months. Twenty-one patients were excluded from the analysis before the first 12 month revision: of these, 13 patients had severe PD and a contralateral subthalamotomy was considered medically necessary within the first year after the initial surgery and these patients entered into a different protocol while another eight patients required a pallidotomy to treat hemichorea-ballism that developed after the first STN lesion (see below). One patient committed suicide 18 months after surgery.

\section{"Off" medication state}

There was a significant reduction in the "off" UPDRS-III at 12 (50\%), 24 (30\%) and 36 months (18\%) postoperatively (fig 3). There was a significant improvement in the cardinal features (bradykinesia, rigidity and tremor) contralateral to the lesion and a mild and non-persistent effect ipsilaterally (table 2). The 
Table 2 Effect of subthalamotomy on the cardinal features of Parkinson's disease

\begin{tabular}{lclcc}
\hline Cardinal sign & Preop & $\mathbf{1 2}$ months & $\mathbf{2 4}$ months & +36 months \\
\hline "Off” motor state & & & & \\
$\quad$ Rigidity (ipsilateral) & $4.6(1.23)$ & $4.6(1.23)$ & $4.8(1.24)$ & $5.8(1.50)$ \\
Rigidity (contralateral) & $6.1(1.30)$ & $1.8(1.04)^{* *}$ & $2.7(1.00)^{* *}$ & $3.7(1.53)^{* *}$ \\
Bradykinesia (ipsilateral) & $10.4(2.66)$ & $9.0(2.46)$ & $10.7(2.98)$ & $12.4(2.56)$ \\
Bradykinesia (contralateral) & $13.4(2.32)$ & $4.6(1.86)^{* *}$ & $6.1(1.52)^{* *}$ & $8.1(2.59)^{* *}$ \\
Tremor (ipsilateral) & $3.7(2.55)$ & $3.4(2.54)$ & $5.1(2.53)$ & $5.7(2.87)$ \\
Tremor (contralateral) & $5.5(3.13)$ & $1.4(1.4)^{* *}$ & $2.4(1.77)^{* *}$ & $3.3(2.09)^{* *}$ \\
“On” motor state & & & & \\
Rigidity (ipsilateral) & $1.9(1.02)$ & $1.6(0.94)$ & $2.0(0.94)$ & $2.6(1.22)$ \\
Rigidity (contralateral) & $2.9(1.27)$ & $0.3(0.72)^{* *}$ & $0.8(0.84)^{* *}$ & $1.5(1.19)^{* *}$ \\
Bradykinesia (ipsilateral) & $4.6(1.79)$ & $3.6(1.69)$ & $4.7(1.93)$ & $5.5(2.43)$ \\
Bradykinesia (contralateral) & $6.5(2.00)$ & $1.1(1.47)^{* *}$ & $2.0(1.48)^{* *}$ & $3.6(1.99)^{* *}$ \\
Tremor (ipsilateral) & $1.2(1.33)$ & $1.0(1.15)$ & $1.7(1.47)$ & $2.3(2.17)^{* *}$ \\
Tremor (contralateral) & $2.2(1.98)$ & $0.2(0.54)^{* *}$ & $0.5(0.93)^{* *}$ & $1.0(1.20)^{*}$ \\
\hline
\end{tabular}

Data presented as mean (SD).

${ }^{*} p<0.05$, ${ }^{* *} p<0.01$ between preoperative (Preop) compared with 12,24 and +36 months postoperatively.

UPDRS-III at 36 months increased by $36.6 \%$ compared with the 12 month postoperative value, revealing a difference $(p<0.01)$ due to higher (60 vs 38 points) scores at 36 compared with 12 months. The increment in the UPDRS-III at 3 years (fig 3 ) was mainly due to a significant increase in the item scores for the ipsilateral side (fig 4C) and a moderate (non-significant) increase in the axial items on the scale (fig 4E). The daily dose of levodopa was reduced by $45 \%(p<0.001), 36 \%(p<0.001)$ and $28 \%(p<0.05)$ at 1,2 and 3 years (fig 3$)$ compared with baseline.
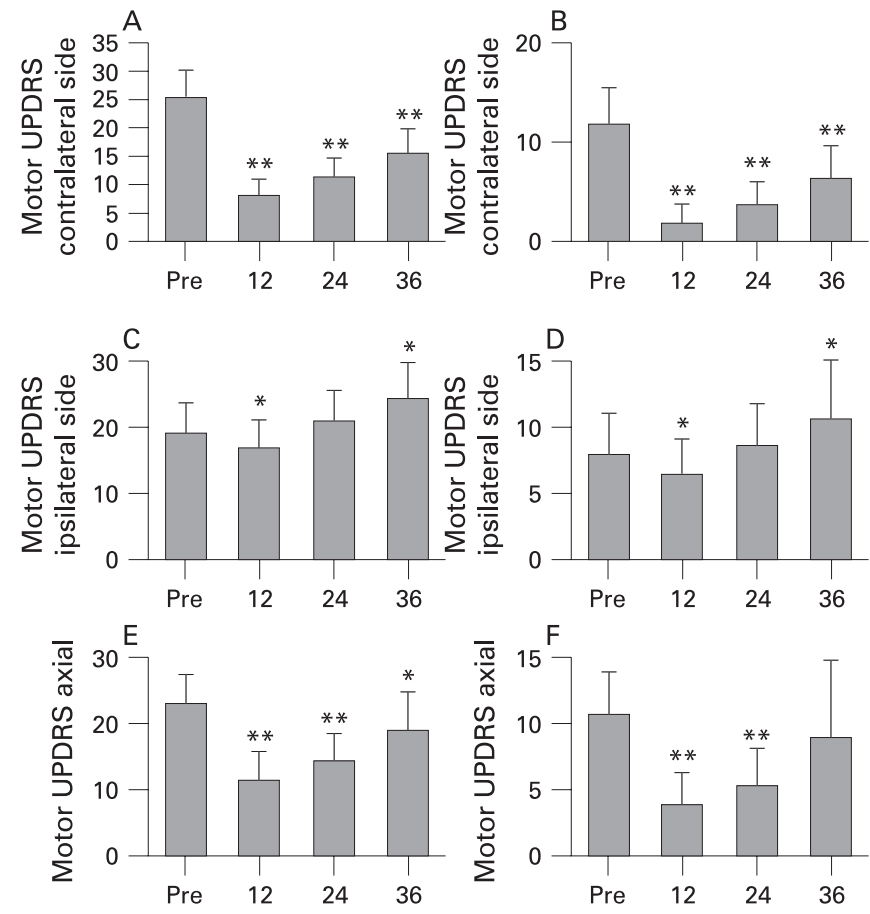

Time (months)

Figure 4 Evolution of the Unified Parkinson's Disease Rating Scale (UPDRS) motor scores on the side contralateral to the subthalamic nucleus lesion, the ipsilateral side and axial signs (items Nos 18-20, 22, 27-31 of the UPDRSm) in the "off" $(A, C, E)$ and "on" (B, D, F) motor states. The anti-parkinsonian effect persisted on the contralateral side but a marked worsening of ipsilateral signs and a return towards the baseline score for axial features is seen. ${ }^{*} p<0.05$, ${ }^{* *} p<0.01$ between preoperative (Pre) compared with 12, 24 and +36 months postoperatively.

\section{"On" medication state}

The UPDRS-III in the "on" motor state was significantly reduced at 12 and 24 months postoperatively (fig 3). The improvement in the "on" was mainly due to a reduction in cardinal features in the contralateral side and axial signs (table 2; fig 4B, 4F). There was a worsening in the UPDRS-III score after 3 years which was no longer different from the baseline evaluation. Comparison of the UPDRS-III between 12 and 36 months showed a significant difference $(p<0.05)$ owing to
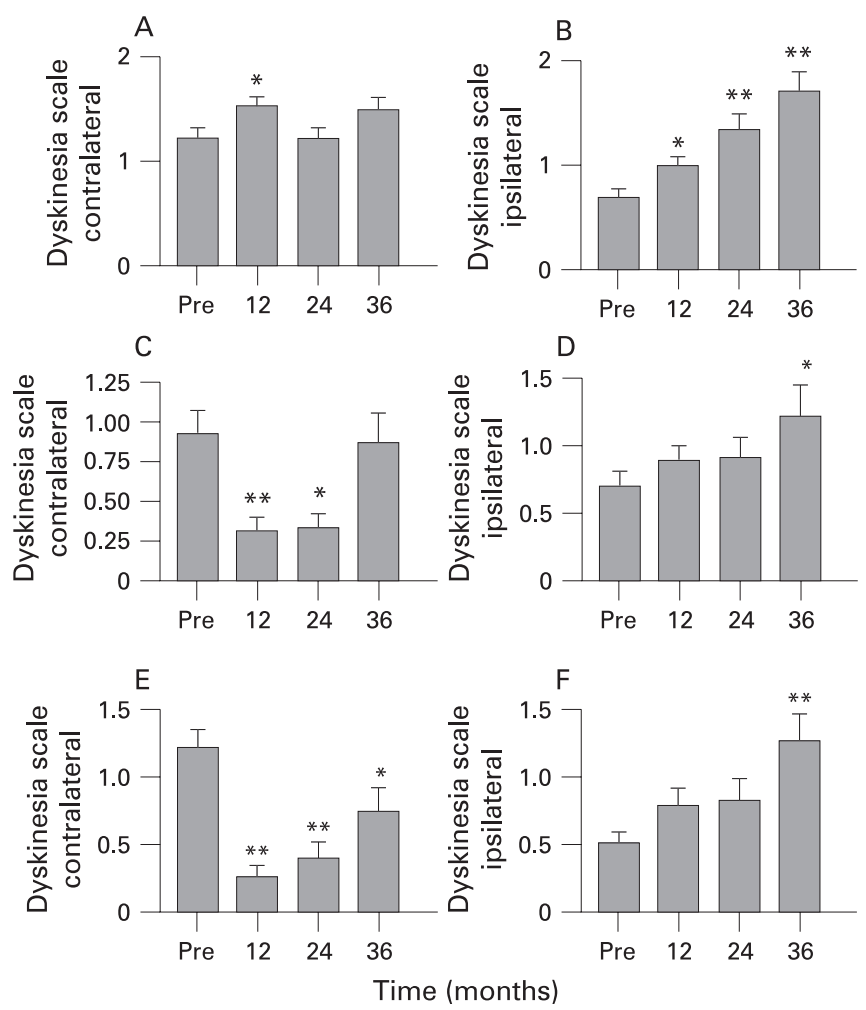

Figure 5 Change in levodopa induced dyskinesias throughout evolution. There was a modest increase in "peak dose" dyskinesias (A) and reduction in diphasic dyskinesias $(C)$ and "off" period dystonia $(E)$ in the first 24 months postoperatively. On the non-operated side, all three subtypes of levodopa induced dyskinesias increased steadily over the study period $(B, D, F) .{ }^{*} p<0.05,{ }^{*} p<0.01$ between preoperative (Pre) compared with 12,24 and +36 months postoperatively. 
higher scores at 36 months. This deterioration at 3 years in the "on" score was related to progressive worsening of the ipsilateral and axial features (fig 4D, 4F) of the UPDRS-III.

\section{Levodopa induced dyskinesias}

Figure 5 summarises the evolution of levodopa induced dyskinesias (LIDs) after surgery for all 68 patients. Preoperatively, patients exhibited more severe LIDs on the operated side, as expected, as subthalamotomy was performed on the most severe side. Peak dose dyskinesias increased on the side contralateral to the lesion during the first postoperative year (fig 5A) but there was no other significant change. In the side ipsilateral to the lesion, dyskinesias progressively increased over the 36 months of follow-up (fig 5B). Diphasic dyskinesias and "off" period dystonia significantly improved $(p<0.01)$ contralateral to the lesions at 12 and 24 months but had increased after 36 months post-surgery. This result was influenced by three patients in whom the control of dyskinesias on the operated side had worsened considerably by the end of the study. On the side corresponding to the non-operated hemisphere, diphasic dyskinesias and "off" dystonia increased steadily from the time of surgery (fig $5 \mathrm{~B}, 5 \mathrm{D}, 5 \mathrm{~F}$ ).

\section{Cognitive functions}

In the 33 patients assessed neuropsychologically, there were no significant changes in the tests with regard to baseline values when they were evaluated 24 months after surgery. Thus no difference was found in general cognition on the Mini-Mental State Examination (mean score 28 (1.5) vs 29.3 (2.3)), visuoconstructive functions (mean score 20 vs 22) and frontal assessment battery (mean score 15.6 (2.6) vs 14.1 (1.4)). Verbal fluency was reduced preoperatively (with respect to control subjects) and improved but remained below the normal range (mean score in control subjects 11 (3.1) vs 16.2 (4.3) in patients preoperatively and 14.0 (4.1) postoperatively). Depression improved moderately, as was shown by a change in the Hamilton scale (mean score 22 (5.9) vs 15 (7.5)).

\section{Complications}

Adverse events were encountered in 20 of 89 patients who underwent surgical procedures. Clinically relevant dyskinesias induced by surgery were present in 14 patients and are described in more detail below. Other complications consisted of transient or mild dysarthria in four (4.4\%), infection of the scalp incision in three patients $(3.3 \%)$, epileptic seizure in two patients $(2.2 \%)$

Table 3 Comparison of preoperative demographic features of patients with $(+)$ and without $(-)$ severe hemichorea-ballism (HCB) after subthalamotomy

\begin{tabular}{lcc}
\hline Patients & HCB $+(\mathbf{n}=\mathbf{1 4})$ & HCB - (n = 61) \\
\hline Age (years) & $53.7(38-68)$ & $56.8(40-73)$ \\
Sex (men/women) & $14 / 0$ & $54 / 7$ \\
Disease duration (years) & $9.5(5-13)$ & $10.7(5-27)$ \\
Levodopa daily dose (mg) & $1187.5(800-1800)$ & $1075.8(600-1800)$ \\
L-dopa (doses/day) & $5.3(3-8)$ & $6.5(4-8)$ \\
Hoehn and Yahr & II - IV & II - IV \\
UPDRS-m† "off" & $61.2(38-71)$ & $66.5(35-83)$ \\
UPDRS-m "on" & $27.3(21-41)$ & $29.7(7-40)$ \\
Dyskinesia score (0-4) & $2.7(2-4)$ & $1.3(0-3)^{*}$ \\
\hline
\end{tabular}

Data presented as mean (range) or number.

fUPDRS-m, Unified Parkinson Disease Rating Scale, motor section (part III), scored in the "off" and "on" motor states.

${ }^{*} \mathrm{p}<0.05$. and asymptomatic bleeding along the recording track trajectory in three patients (3.3\%). None of these problems was associated with disability or permanent deficit.

Dyskinesias were observed within the first $24 \mathrm{~h}$ in 52 patients (58.4\%) before any antiparkinsonian medication was reintroduced. In all cases the dyskinesias were contralateral to the lesion. These were of focal distribution (usually the leg or foot) and of mild intensity and did not impair motor control in the large majority of patients, evolving towards self-resolution over the subsequent 4-12 weeks. However, in $14(15 \%)$ patients the dyskinesias persisted. In six of these patients the dyskinesias were focal (mainly affecting the lower limb), mild in intensity (grade I or 2) and did not increase with levodopa, thus inducing no disability. On the other hand, dyskinesias were severe (grade IV) in eight patients ( $9 \%$ ), did not cease after all dopaminergic treatments were halted or even after tetrabenazine and sulpiride were used (three patients) and became a major management problem. In most of these patients, dyskinesias were present by the end of the surgery or began a few hours after the lesion and predominated in the lower limb. In one patient, onset was delayed until 7 days after surgery. In this instance, a second CT repeated after the onset of dyskinesias showed a large area of infarct in the subthalamic region spreading up to the thalamus. In all of these patients there was a sustained and marked improvement in cardinal features. The interval between subthalamotomy and pallidotomy was variable (4 weeks to 2 years). At the beginning, every attempt to manage the dyskinesias pharmacologically was made. Subsequently, the delay was reduced. The hemichorea-ballism was eliminated in all patients by the end of the pallidotomy while the antiparkinsonian benefit previously achieved by subthalamotomy persisted on the operated side. We could not recognise any new adverse neurological event. In any case, the management of clinical features and management details related to hemichoreaballism after subthalamotomy is a special situation which requires expert neurological care. Our full experience of this complication will be reported in the near future.

Comparison of the preoperative demographic features and clinical characteristics of the patients who developed $(n=14)$ severe dyskinesias after subthalamotomy with the subgroup $(n=75)$ who did not, showed no significant differences except for the levodopa induced dyskinesia score, which was higher in the hemichorea-ballism subgroup $(p<0.01)$ (table 3 ). Other putative factors influencing the onset of severe dyskinesias after subthalamotomy, such as the volume and location of the lesion, require a detailed analysis of the images with technology that has only recently become available to the centre.

\section{DISCUSSION}

We have described the clinical effect and long term evolution of patients with advanced PD treated with unilateral subthalamotomy. This series comprised a large number of patients followed for up to 3 years providing a representative sampling of the merit and complications associated with this approach. In our experience, unilateral subthalamotomy induces marked improvement in the cardinal motor features of PD contralateral to the lesion and also, although to a lesser extent, in the axial manifestations of the illness. The ipsilateral effects of the lesions were modest and only reached statistical significance 1 year after operation. Subthalamotomy increased the mean LID score in the side contralateral to the lesion in the first year after surgery but this was not observed subsequently, in contrast with the progressive worsening of dyskinesias observed on the non-operated side (fig 5). This observation has both practical 
and theoretical interest. In practice, it suggests that the presence of mild to moderate LIDs is not an absolute contraindication for subthalamotomy. However, we did exclude from this series patients with severe (ie, grade IV dyskinesia) preoperative LID for whom pallidal surgery may still be a better option. Moreover, patients who developed hemichorea-ballism after subthalamotomy had more severe LIDs preoperatively. For the time being it would thus appear more prudent to continue avoiding subthalamotomy in patients with $\mathrm{PD}$ with moderate to severe LIDs. From a theoretical point of view, it is extremely interesting that LIDs were not increased contralateral to the lesion while they continued to worsen in the non-operated side. The reduction of LIDs in patients with PD treated with STNDBS is often explained as a result of decreased levodopa intake. ${ }^{26}{ }^{27}$ Our patients also reduced their daily levodopa dose after surgery but this did not preclude a progressive rise in LIDs on the non-operated side. This simple observation strongly argues in favour of functional changes in the basal ganglia of the hemisphere on which the operation was performed which reduces the sensitivity to produce LIDs. We have proposed ${ }^{28}$ that surgery on the STN may have an effect similar to those induced by continuous infusion of dopaminergic agents, which increase the dyskinesia threshold. ${ }^{29}$

We encountered no evidence of cognitive impairment resulting from surgery. This is in keeping with another study focusing on unilateral lesions ${ }^{30}$ as well as our own experience. ${ }^{25}$ In the larger series reported here, verbal fluency was actually slightly improved. However, we remain concerned about the possibility that STN lesions, particularly bilateral lesions, could aggravate dysarthria in patients with severe PD. ${ }^{17}$ This concern remains an open question in need of further study. Indeed, the role of bilateral and simultaneous lesion of the STN requires careful evaluation.

From the above it may be concluded that the principal antiparkinsonian benefits of unilateral subthalamotomy persist for up to 3 years postoperatively. However, disease progression leads to increased disability both in the "off" and "on" medication states. Specifically, over the 3 years of follow-up, patients evidenced a progressive deterioration in motor function with a marked worsening of axial and ipsilateral motor signs. Similar findings have been reported, first for pallidotomy ${ }^{31}$ and more recently for bilateral DBS of the globus pallidus pars interna and STN. ${ }^{32}{ }^{33}$ The clinical usefulness and application of unilateral ablative surgery in PD appears optimal for patients with severe but relatively asymmetrical motor manifestations. In this regard, the timing of surgery plays a critical role. The general approach to date has been to delay the surgery for as long as possible. Our experience suggests that, in carefully selected patients with significant asymmetry, the opposite may in fact be true. In our view, once these patients cannot be well controlled pharmacologically, the earlier surgery is performed the better the outcome is likely to be. Although the rate of dyskinesias after surgery was notable (58.8\%) in the majority of these patients, the evolution of the choreatic movements was benign. In a few patients $(n=8,9 \%)$, the dyskinesias were severe, reaching hemiballistic proportions and requiring a second surgery (pallidotomy) to control them. This second procedure was well tolerated, in keeping with previous reports. ${ }^{345}$ Nevertheless, the incidence of lesion induced dyskinesias is still sobering and potentially dangerous, ${ }^{15}$ particularly in patients with advanced PD. There are probably predisposing clinical features and surgery specific factors that account for the development of dyskinesias after subthalamotomy, most of which remain to be determined. To date, we have only been able to note that patients who developed hemichoreaballism had significantly higher dyskinesia scores preoperatively. This suggests a predisposition or increased sensitivity to developing dyskinesias acutely, acting at the level of the STN. Intuitively, we can surmise that the location and volume of the lesion may be a determining factor. In our experience, the volume of the lesion does not appear to be critical, as both large and small lesions have been associated with severe dyskinesias. The location of the lesion may be more relevant. Lozano ${ }^{36}$ suggests that subthalamotomy does not generally provoke dyskinesias because the lesion impinges on the thalamic fasciculus running dorsal to the STN, thus producing a pallidotomy-like effect. A similar interpretation has been proposed for the antidyskinetic effect of STN-DBS. ${ }^{37}$ According to such an interpretation, larger lesions which typically reach the zona incerta and Forel's field dorsal to the STN should be associated with less dyskinesias. Our experience with unilateral and bilateral subthalamotomies does not support this explanation. We and others have encountered severe and persistent hemichorea-ballism in patients with lesions clearly affecting Forel's field, ${ }^{13}{ }^{17}$ and even invading the ventral thalamus. ${ }^{38}{ }^{39}$ Alternatively, we suggest that some lesions may affect sub-regions of the STN which are critically important in the induction of chorea-ballism.

In conclusion, the results presented here confirm and expand previous reports ${ }^{13-17}$ indicating that unilateral subthalamotomy has a robust antiparkinsonian effect and may be performed with reasonable safety in $\mathrm{PD}$. Clinical outcomes could potentially be improved with further surgical refinements aimed at reducing or avoiding lesion associated dyskinesias. The timing of surgery may also require modification to maximise the period of adequate control.

Acknowledgements: The unfailing and generous support of Drs Julian Alvarez and Emilio Villa, director and vice-director of the CIREN, throughout this project, is greatly appreciated. Cristina Calvo, Esperanza Segurado and Ruth Grace edited and corrected the article for publication.

Competing interests: None.

Ethics approval: The study was approved by the institutional scientific committee and the Cuban National Ethics Committee.

\section{REFERENCES}

1. DeLong MR. Primate models of movement disorders of basal ganglia origin. Trends Neurosci 1990;13:281-5.

2. Vila M, Levy R, Herrero MT, et al. Consequences of nigrostriatal denervation on the functioning of the basal ganglia in human and nonhuman primates: an in situ hybridization study of cytochrome oxidase subunit I mRNA. J Neurosci 1997; 17:765-73

3. Hutchison WD, Allan RJ, Opitz $\mathrm{H}$, et al. Neurophysiological identification of the subthalamic nucleus in surgery for Parkinson's disease. Ann Neurol 1998;44:622-8.

4. Rodriguez-Oroz MC, Rodriguez M, Guridi J, et al. The subthalamic nucleus in Parkinson's disease: somatotopic organization and physiological characteristics. Brain 2001;124:1777-90

5. Bergman H, Wichmann T, DeLong MR. Reversal of experimental parkinsonism by lesions of the subthalamic nucleus. Science 1990;249:1436-8.

6. Aziz TZ, Peggs D, Sambrook MA, et al. Lesion of subthalamic nucleus for the alleviation of 1-methyl-4-phenyl; 1,2,3,6-tetrahydropyridine (MPTP)-induced parkinsonism in the primate. Mov Disord 1991;6:288-93.

7. Guridi J, Herrero MT, Luquin MR, et al. Subthalamotomy in parkinsonian monkeys Behavioural and biochemical analysis. Brain 1996;119:1717-27.

8. Guridi J, Herrero MT, Luquin MR, et al. The subthalamic nucleus: a new possible stereotaxic target for Parkinson's disease. Mov Disord 1993;8:421-9.

9. Dierssen G, Bergmann L, Gioino L, et al. Hemiballism following surgery for Parkinson's disease. Arch Neurol 1961;5:627-37.

10. Guridi J, Obeso JA. The subthalamic nucleus, hemiballismus and Parkinson's disease: reappraisal of a neurosurgical dogma. Brain 2001;124:5-19.

11. Gill SS, Heywood P. Bilateral dorsolateral subthalamotomy for advanced Parkinson's disease. Lancet 1997;350:1224.

12. Obeso JA, Alvarez L, Macias R, et al. Lesion of the subthalamic nucleus (STN) in Parkinson's disease (PD). Neurology 1997;48(Suppl 3):138A 
13. Alvarez L, Macias R, Guridi J, et al. Dorsal subthalamotomy for Parkinson's disease. Mov Disord 2001;16:72-8.

14. Patel NK, Heywood P, O'Sullivan K, et al. Unilateral subthalamotomy in the treatment of Parkinson's disease. Brain 2003:126:1136-45.

15. Su PC, Tseng HM, Liu HM, et al. Subthalamotomy for advanced Parkinson disease. $J$ Neurosurg 2002; 97:598-606.

16. Vilela F, da Silva DJ. Unilateral subthalamic nucleus lesioning: a safe and effective treatment for Parkinson's disease. Arq Neuropsiquiatr 2002;60:935-48.

17. Alvarez L, Macias R, Lopez G, et al. Bilateral subthalamotomy in Parkinson's disease: initial and long-term response. Brain 2005:128:570-83.

18. Hughes AJ, Daniel SE, Kilford L, et al. Accuracy of clinical diagnosis of idiopathic Parkinson's disease: a clinico-pathological study of 100 cases. J Neurol Neurosurg Psychiatry 1992;55:181-4.

19. Lopez-Flores G, Morales JM, Tejeiro-Amador J, et al. Anatomic and neurophysiological methods for the targeting and lesioning of the subthalamic nucleus: Cuban experience and review. Neurosurgery 2002;52:817-29.

20. Macias R, Teijeiro R, Torres A, et al. Electrophysiological targeting in stereotaxic surgery for Parkinson's disease. Adv Neurol 1997:74:175-82.

21. Teijeiro J, Macias R, Morales JM, et al. Personal-computer-based system for threedimensional anatomic-physiological correlations during stereotactic functional neurosurgery. Stereotact Funct Neurosurg 2000;75:176-87.

22. Carballo-Barreda M, Rodríguez-Rojas R, Lopez G, et al. Sistema tridimensional de planeamiento quirúrgico para PC. Ingeniería Electrónica Automática y Comunicaciones 2005;26:19-23

23. Langston JW, Widner $\mathrm{H}$, Goetz CG, et al. Core assessment program for intracerebral transplantations (CAPIT). Mov Disord 1992:7:2-13.

24. Goetz CG, Stebbins GT, Shale HM, et al. Utility of an objective dyskinesia rating scale for Parkinson's disease: inter- and intrarater reliability assessment. Mov Disord 1994:9:390-4.

25. Bickel S, Fernandez C, Alvarez L, et al. Cognitive and neuropsychiatric effects of subthalamotomy for Parkinson disease. Parkinsonism Relat Disord (in press).
26. Fraix V, Pollak P, Van Blercom N, et al. Effect of subthalamic nucleus stimulation on levodopa-induced dyskinesia in Parkinson's disease. Neurology 2000;55:1921-3.

27. Vingerhoest FJG, Villemure JG, Temperli P, et al. Subthalamic DBS replaces levodopa in Parkinson's patients. Two years follow up. Neurology 2002:58:396-401.

28. Guridi J, Obeso JA, Rodriguez-Oroz MC, et al. L-Dopa-induced dyskinesia and stereotactic surgery for Parkinson's disease. Neurosurgery 2008;62:311-25.

29. Mouradian MM, Juncos JL, Fabbrini G, et al. Motor fluctuations in Parkinson's disease: central pathophysiological mechanisms Part II. Ann Neurol 1988;24:372-8.

30. McCarter RJ, Walton NH, Rowan AF, et al. Cognitive functioning after subthalamic nucleotomy for refractory Parkinson's disease. J Neurol Neurosurg Psychiatry 2000;69:60-6.

31. Fine $\mathbf{J}$, Duff $\mathrm{J}$, Chen $\mathrm{R}$, et al. Long-term follow-up of unilateral pallidotomy in advanced Parkinson's disease. N Engl J Med 2000;342:1708-14.

32. Krack P, Batir A, Van Blercom N, et al. Five-year follow-up of bilateral stimulation of the subthalamic nucleus in advanced Parkinson's disease. N Engl J Med 2003;349:1925-34.

33. Rodriguez-Oroz MC, Obeso JA, Lang $A E$, et al. Bilateral deep brain stimulation in Parkinson's disease: a multicentre study with 4 years follow-up. Brain 2005; 128:2240-9.

34. Suarez JI, Metman LV, Reich SG, et al. Pallidotomy for hemiballismus: efficacy and characteristics of neuronal activity. Ann Neurol 1997:42:807-11.

35. Vitek JL, Chockkan V, Zhang JY, et al. Neuronal activity in the basal ganglia in patients with generalized dystonia and hemiballismus. Ann Neurol 1999;46:22-35.

36. Lozano AM. The subthalamic nucleus. Myth and opportunities. Mov Disord 2001;16:183-4.

37. Herzog J, Pinsker $M$, Wasner $M$, et al. Stimulation of subthalamic fibre tracts reduces dyskinesias in STN-DBS. Mov Disord 2007;22:679-84.

38. Gioino GG, Dierssen G, Cooper IS. The effect of subcortical lesions on production and alleviation of hemiballistic or hemichoreic movements. J Neurol Sci 1966:3:10-36.

39. Guridi J, Obeso JA. The subthalamic nucleus, hemiballismus and Parkinson's disease: reappraisal of a neurosurgical dogma. Brain 2001:124:5-19.

\section{Access a vast information database with Toll-Free linking}

"Toll-free" linking gives you immediate access to the full text of many of the cited articles in a paper's reference list-FOR FREE. With the support of HighWire's vast journal catalogue, a huge reference library is now open to you. If HighWire hosts the journal, you can view the full text of the referenced article, completely free of charge by following the Free Full Text links in the references. 